NBER WORKING PAPER SERIES

PRECAUTIONARY SAVING IN THE SMALL AND IN THE LARGE

Miles S. Kimball

Working Paper No. 2848

\author{
NATIONAL BUREAU OF ECONOMIC RESEARCH \\ 1050 Massachusetts Avenue \\ Cambridge, MA 02138 \\ February 1989
}

I would like to thank Greg Mankiw, Leslie Young, Lars Hansen, Stephen Zeldes, Phillipe Weil and Ted Bergstrom for helpful discussions, participants in various seminars for insightful comments, and the editor and two anonymous referees for excellent suggestions. This paper is part of NBER's research program in Financial Markets and Monetary Economics. Any opinions expressed are those of the author not those of the National Bureau of Economic Research. 
NBER Working Paper $\$ 2848$

February 1989

\section{PRECAUTIONARY SAVING IN THE SMALL AND IN THE LARGE}

\section{ABSTRACT}

The theory of precautionary saving is shown in this paper to be isomorphic to the Arrow-Pratt theory of risk aversion, making possible the application of a large body of knowledge about risk aversion to precautionary saving, and more generally, to the theory of optimal choice under risk. In particular, a measure of the strength of precautionary saving motive analogous to the Arrow-Pratt measure of risk aversion is used to establish a number of new propositions about precautionary saving, and to give a new interpretation of the Dreze-Modigliani substitution effect.

Miles S. Kimball Department of Economics University of Michigan Ann Arbor, MI 48109 


\section{Introduction}

It has been recognized since Bernoulli (1738) that risk aversion can be associated with concavity of utility functions. But it was not until Pratt (1964) and Arrow (1965) that it was recognized that the function $\frac{-v^{\prime \prime}(x)}{v^{\prime}(x)}$-or the related index $\frac{-v^{\prime \prime}(x)}{v^{\prime}(x)} x$-was an excellent measure of risk aversion. Subsequently, the Arrow-Pratt measures of absolute and relative risk aversion have often demonstrated their usefulness in a wide range of both theoretical and empirical studies of behavior under uncertainty. ${ }^{1}$ In addition, the example of the Arrow-Pratt measures of risk aversion has spurred a considerable amount of research on other ways to characterize aspects of risk aversion, such as Ross' (1981) work on a notion of "strongly more risk averse" or Pratt and Zeckhauser's (1987) work on "proper risk aversion."

In the study of precautionary saving, it has been known since Leland (1968) and Sandmo (1970) that precautionary saving in response to risk is associated with convexity of the marginal utility function, or a positive third derivative of a von Neumann-Morgenstern utility function. More generally, Rothschild and Stiglitz (1971) note that if an individual's utility is a function of a control variable $\delta$ and an exogenous random variable $\theta$, so that he or she solves

$$
\max _{\delta} \mathbf{E} V(\theta, \delta)
$$

using the first-order condition

$$
\mathbf{E} \frac{\partial V}{\partial \delta}=\mathbf{0}
$$

then if $\frac{\partial V}{\partial \delta}$ is convex in $\theta$, increases in the variability of $\theta$ will result in increases in the optimal choice of $\delta$. This is analogous to the fact that a concave utility function indicates risk aversion. However, unlike the theory of risk aversion, which lays out in considerable detail the determinants of the magnitude of the effects of risk on expected utility, the theory of the optimal response of decision variables to risk (which includes precautionary saving as a subcase) has not hitherto said much about the magnitude of these responses. Fortunately, as will be shown, this is an easily remedied deficiency, since a straightforward reinterpretation of the mathematical theory yields an equally powerful theory of the optimal response of decision variables to risk-and in particular, a theory of precautionary saving closely analogous to the Arrow-Pratt theory of risk aversion.

Mathematically, the Arrow-Pratt index $-v^{\prime \prime}(x) / v^{\prime}(x)=a(x)$ is a good measure of risk aversion because given two utility functions $v_{1}(x)$ and $v_{2}(x)$, if $a_{1}(x)>a_{2}(x)$ for all $x$, then $v_{1}(x)=g\left(v_{2}(x)\right.$ ) where $g(\cdot)$ is a monotonically increasing, concave function. ${ }^{2}$ If $v_{1}$ is thus a concave or "risk averse"

1 The References include many papers in this category.

2 See Pratt (1964) for a proof. 
transformation of $v_{2}$, then it can readily be shown that $v_{1}$ implies more risk averse behavior than $v_{2}$.

Analogously, as long as the cross-partial $\frac{\partial^{2} V(\theta, \delta)}{\partial \theta \partial \delta}$ is uniformly positive or uniformly negative, the index $-\frac{\partial^{3} V(\theta, \delta)}{\partial \theta^{2} \partial \delta} / \frac{\partial^{2} V(\theta, \delta)}{\partial \theta \partial \delta}=\eta(\theta, \delta)$ is a good measure of the sensitivity of the optimal choice of $\delta$ to risk because (by the same mathematical result used above) given two indirect utility functions $V_{1}(\theta, \delta)$ and $V_{2}(\theta, \delta)$ and a fixed initial value of $\delta$, if $\eta_{1}(\theta, \delta)>\eta_{2}(\theta, \delta)$ for all $\theta$, then $\frac{\partial V_{1}(\theta, \delta)}{\partial \delta}=h\left(\frac{\partial V_{3}(\theta, \delta)}{\partial \delta}\right)$ where $h(\cdot)$ is monotonically increasing and concave if $\frac{\partial^{2} V(\theta, \delta)}{\partial \theta \partial \delta}>0$ but convex if $\frac{\partial^{2} V(\theta, \delta)}{\partial \theta \partial \delta}<0$. If $\frac{\partial V_{1}(\theta, \delta)}{\partial \delta}$ is thus a concave or convex transformation of $\frac{\partial V_{2}(\theta, \delta)}{\partial \delta}$, then in certain important senses detailed below, the indirect utility function $V_{1}$ implies a different degree of sensitivity of the optimal choice of $\delta$ to risk than the indirect utility function $V_{2}$.

If we give the name "prudence" to the sensitivity of the optimal choice of a decision variable to risk, then $\eta(\theta, \delta)$ is a measure of "absolute prudence," and $\theta \eta(\theta, \delta)$ is a measure of "relative prudence," just as $a(x)$ is a measure of absolute risk aversion and $x a(x)$ is a measure of relative risk aversion. The term "prudence" is meant to suggest the propensity to prepare and forearm oneself in the face of uncertainty, in contrast to "risk aversion," which is how much one dislikes uncertainty and would turn away from uncertainty if one could. ${ }^{3}$

Though the analogy between the theory of risk aversion and the theory of the optimal response of decision variables to risk is quite general, concreteness is especially important in illustrating a technique of reinterpretation. The determination of precautionary saving is an example of the effect of risk on a decision variable that is not only simple, but also one that has considerable importance in its own right. Therefore, after a few remarks on a general plane in Section II, establishing the usefulness of "prudence" as a measure of the sensitivity of choices to risk, we will turn to a deeper investigation of precautionary saving. ${ }^{4}$ Section III establishes the formal similarity between the theory of precautionary saving in particular and the theory of risk aversion. Section IV applies the most basic results about risk aversion to precautionary saving. Section $V$ explores the consequences of decreasing, increasing or constant absolute prudence in the context of precautionary saving, including the effects of uncertainty on the marginal propensity to consume out of wealth. Section VI briefly considers the consequences of facing more than one risk at a time. Section VII uses the concept of "prudence" to provide a novel interpretation of the Drèze-Modigliani (1972) substitution effect as the consequence of the precautionary saving motive being stronger than risk aversion in

3 In different contexts, "prudence" will have different meanings. In the paradigmatic example of the consumptionsaving decision under uncertainty, "prudence" represents the intensity of the precautionary saving motive.

4 Kimball (1988a) offers another, quite different, example of how the mathematical theory of risk aversion can be used to study optimal decisions under risk. 
the case of decreasing absolute risk aversion and weaker than risk aversion in the case of increasing absolute risk aversion. Section VIII concludes the paper.

\section{Prudence as a Measure of the Sensitivity of a Decision Variable to Risk}

Four of the basic results in the theory of risk aversion involving risk premia can be applied readily to any model of choice under uncertainty that falls under Rothschild and Stiglitz' (1971) general description. In order to state these results we must first define two concepts analogous to risk premia.

Let a probability distribution for $\theta$ be analyzed into $\theta=\theta_{0}+\bar{\theta}$ where $\theta_{0}$ is a certain quantity and $\bar{\theta}$ is a random variable. Then we will call $\psi$ an "equivalent precautionary premium" for $\tilde{\theta}$ if it satisfies

$$
\mathbf{E} \frac{\partial V\left(\theta_{0}+\tilde{\theta}, \delta_{1}\right)}{\partial \delta}=\frac{\partial V\left(\theta_{0}-\psi, \delta_{1}\right)}{\partial \delta}
$$

for some $\delta_{1}$. If both sides of (1) equal zero, then $\delta_{1}$ satisfies the first order conditions for an optimum for two different distributions of $\theta$ : for $\theta$ equal to $\theta_{0}-\psi$ with certainty, and for $\theta$ equal to the random variable $\theta_{0}+\tilde{\theta}$. If these first order conditions are also sufficient for an optimum, then the equivalent precautionary premium $\psi$ is the certain reduction in $\theta$ from the initial value $\theta_{0}$ that has the same effect on the optimal value of the decision variable as the addition of the random variable $\vec{\theta} ;$ in both cases the optimal value of $\delta$ changes from $\delta_{0}=\arg \max _{\delta} V\left(\theta_{0}, \delta\right)$ to $\delta_{1}$.

Similarly, we will call $\psi^{*}$ a "compensating precautionary premium" ${ }^{5}$ for $\tilde{\theta}$ if it satisfies

$$
\mathbf{E} \frac{\partial V\left(\theta_{0}+\bar{\theta}+\psi^{*}, \delta_{0}\right)}{\partial \delta}=\frac{\partial V\left(\theta_{0}, \delta_{0}\right)}{\partial \delta}
$$

If both sides of (2) equal zero-as they will be if $\delta_{0}$ is defined as at the end of the previous paragraph-and these first order conditions are sufficient for an optimum, then the compensating precautionary premium $\psi^{*}$ is the shift in the distribution of $\theta$ that compensates for the effect of the random variable $\tilde{\theta}$ on the optimal value of $\delta^{6}$

The parallel between these precautionary premia and risk premia can be shown more clearly by Table 1. Pratt's (1964) notation $\pi$ is used to denote an equivalent risk premium and $\pi^{*}$ to denote

5 See Pope and Chavas (1985) for a discussion of the distinction between equivalent and compensating risk premia, which are analogous to equivalent and compensating variations under certainty. Pratt (1964) refers to this distinction in passing, noting that the "asking price" price of a risk (the equivalent risk premium) is different from the "bid price" of a risk (the compensating risk premium). He refers to the compensating risk premium as the "bid price" of a risk.

6 Compensation for an effect on a decision variable should not be confused with welfare compensation. The two often do not coincide, as can be seen, for example, in Section VII on the Dréze-Modigliani substitution effect. 
a compensating risk premium. ${ }^{7}$ Table 1 shows the definitions of the precautionary premia for both the special case of precautionary saving, which will be addressed in the succeeding sections, and for the general case.

\section{Table 1}

\section{Comparison of Risk and Precautionary Premia}

\section{Equivalent Risk Premium $\pi$}

$$
\mathbf{E} v(x+\tilde{z})=v(x-\pi(\tilde{z}, x))
$$

Equivalent Precautionary Premium $\psi$

$$
\begin{array}{r}
(\text { precautionary saving case }) \\
\mathbf{E} v^{\prime}(s+\bar{y})=v^{\prime}(s-\psi(\tilde{y}, s)) \\
(\text { general case }) \\
\mathbf{E} \frac{\partial V\left(\theta_{0}+\bar{\theta}, \delta\right)}{\partial \delta}=\frac{\partial V\left(\theta_{0}-\psi\left(\tilde{\theta}, \theta_{0}, \delta\right), \delta\right)}{\partial \delta}
\end{array}
$$

\section{Compensating Risk Premium $\pi^{*}$}

$\mathbf{E} v\left(x+\tilde{z}+\pi^{*}(\bar{z}, x)\right)=v(x)$

Compensating Precautionary Premium $\psi^{*}$ (precautionary saving case)

$\mathbf{E} v^{\prime}\left(s+\bar{y}+\psi^{*}(\bar{y}, s)\right)=v^{\prime}(s)$

(general case)

$\mathbf{E} \frac{\partial V\left(\theta_{0}+\tilde{\theta}+\psi^{*}\left(\tilde{\theta}, \theta_{0}, \delta\right), \delta\right)}{\partial \delta}=\frac{\partial V\left(\theta_{0}, \delta\right)}{\partial \delta}$

Note: In the arguments of $\pi, \pi^{*}, \psi$, and $\psi^{*}$, the symbols $\tilde{z}, \tilde{y}$ and $\tilde{\theta}$ represent the entire distributions of $\tilde{z}, \tilde{y}$ and $\tilde{\theta}$, not particular realizations.

The only difference between the definition of a precautionary premium and the definition of the corresponding risk premium is in the substitution of marginal utility with respect to the decision variable for total utility. Furthermore, given our assumption that $\frac{\partial^{2} V(\theta, \delta)}{\partial \theta \partial \delta}$ is either uniformly positive or uniformly negative over the relevant region, either $\frac{\partial V(\theta, \delta)}{\partial \delta}$ or $-\frac{\partial V(\theta, \delta)}{\partial \delta}$, considered as a function of $\theta$, is a monotonically increasing function to which theorems about monotonically increasing utility functions can be applied. Since changing the sign of both sides of the definition of a precautionary premium leaves an equivalent expression, the substitution of $\frac{\partial V}{\partial \delta}$ or $-\frac{\partial V}{\partial \delta}$ for $v$ and the substitution of $\psi$ and $\psi^{*}$ for $\pi$ and $\pi^{*}$ describes a complete isomorphism between statements about risk premia and statements about precautionary premia.

Two of the most important theorems about equivalent risk premia can be found in John Pratt's (1964) brilliant article "Risk Aversion in the Small and in the Large." The "risk aversion in the

7 Throughout the paper, all formulas involving $\pi, \pi *, \psi$ and $\psi^{*}$ apply even when the risks involved do not have zero means, unless stated otherwise. However, when $\bar{z}$ or $\tilde{y}$ have non-zero means, $\pi, \pi *, \psi$ and $\psi^{*}$ must be interpreted as minus certainty equivalents or minus certainty compensations, rather than as premia. 
small" of Pratt's title refers to the result that for a small mean-zero uncertainty $\bar{z}$, the equivalent risk premium is

$$
\pi(\bar{z}, x)=\frac{-v^{\prime \prime}(x)}{v^{\prime}(x)} \frac{\sigma_{z}^{2}}{2}+o\left(\sigma_{z}^{2}\right)=a(x) \frac{\sigma_{z}^{2}}{2}+o\left(\sigma_{z}^{2}\right),
$$

where $o\left(\sigma_{z}^{2}\right)$ denotes a quantity that approaches zero faster than $\sigma_{z}^{2}$ as $\sigma_{z}^{2} \rightarrow 0$. In an even more striking result, Pratt demonstrates that absolute risk aversion also indicates the intensity of "risk aversion in the large" since given two utility functions $v_{1}$ and $v_{2}$, if $v_{1}$ always has a higher measure of absolute risk aversion-that is, $a_{1}(x)=\frac{-v_{2}^{\prime \prime}(x)}{v_{1}^{\prime}(x)}>\frac{-v_{2}^{\prime \prime}(x)}{v_{2}^{\prime}(x)}=a_{2}(x)$ for all $x$-then the equivalent risk premium will always be greater for $v_{1}$ than for $v_{2}\left(\pi_{1}(\tilde{z}, x)>\pi_{2}(\bar{z}, x)\right.$ for all $x$ and $\left.\tilde{z}\right)$. This is a result of the fact we noted above that if $a_{1}(x)>a_{2}(x)$ for all $x$ then $v_{1}(x)$ is a concave transformation of $v_{2}(\boldsymbol{x})$.

These two results are readily translated into the language of optimal choice under risk. For a small mean-zero uncertainty, $\tilde{\theta}$, the equivalent precautionary premium is

$$
\psi\left(\tilde{\theta}, \theta_{0}, \delta\right)=-\frac{\frac{\partial^{3} V(\theta, \delta)}{\partial \theta^{2} \partial \delta}}{\frac{\partial^{2} V(\theta, \delta)}{\partial \theta \partial \delta}} \frac{\sigma_{\theta}^{2}}{2}+o\left(\sigma_{\theta}^{2}\right)=\eta(\theta, \delta) \frac{\sigma_{\theta}^{2}}{2}+o\left(\sigma_{\theta}^{2}\right) .
$$

"In the large," if given two indirect utility functions $V_{1}$ and $V_{2}$ and a fixed initial value of $\delta$, if $V_{1}$ has a globally higher measure of absolute prudence than $V_{2}$-that is,

$$
\eta_{1}(\theta, \delta)=-\frac{\frac{\partial^{3} V_{1}(\theta, \delta)}{\partial \theta^{2} \partial \delta}}{\frac{\partial^{2} V_{1}(\theta, \delta)}{\partial \theta \partial \delta}}>-\frac{\frac{\partial^{3} V_{2}(\theta, \delta)}{\partial \theta^{2} \partial \delta}}{\frac{\partial^{2} V_{2}(\theta, \delta)}{\partial \theta \partial \delta}}=\eta_{2}(\theta, \delta)
$$

for all $\theta$-then the equivalent precautionary premium will be greater for $V_{1}$ than for $V_{2}$ $\left(\psi_{1}\left(\tilde{\theta}, \theta_{0}, \delta\right)>\psi_{2}\left(\tilde{\theta}, \theta_{0}, \delta\right)\right.$ for all $\theta_{0}$ and $\left.\tilde{\theta}\right) .^{8}$

Pratt (1964) mentions the concept of a compensating risk premium only in passing, ${ }^{9}$ but the statements above apply to compensating risk premia and precautionary premia as well as to equivalent risk premia and precautionary premia. Appendix A shows that $f(x)=x-\pi(\bar{z}, x)$ and $g(x)=x+\pi^{*}(\tilde{z}, x)$ are mutual inverses, ${ }^{10}$ so that for a small zero-mean risk $\tilde{z}$,

$$
\begin{aligned}
\pi^{*}(\tilde{z}, x) & =\pi\left(\tilde{z}, x+\pi^{*}(\tilde{z}, x)\right) \\
& \left.=a\left(x+\pi^{*}(\tilde{z}, x)\right) \frac{\sigma_{z}^{2}}{2}+o\left(\sigma_{z}^{2}\right)\right) \\
& =a(x) \frac{\sigma_{z}^{2}}{2}+o\left(\sigma_{z}^{2}\right),
\end{aligned}
$$

8 This is true both when the cross partials $\frac{\delta^{2} V(1, \delta)}{\partial \theta \delta \delta}$ are positive and when they are negative. However, it is essential that the cross partials have the same sign for both $V_{1}$ and $V_{2}$.

9 See footnote.

10 These are ordinary point-valued functions, since as long as the utility function is monotonically increasing, there is at most one possible value for a risk premium. 
as long as absolute risk aversion $a(x)$ is continuous. Furthermore, the fact that $f(x)=x-\pi(\tilde{z}, x)$ and $g(x)=x+\pi^{*}(\tilde{z}, x)$ are mutual inverses implies the following lemma:

Lemma: Let each utility function be strictly increasing and continuous and let $\pi_{1}(\tilde{z}, x), \pi_{1}^{*}(\tilde{z}, x)$, $\pi_{2}(z, x)$ and $\pi_{2}^{*}(\tilde{z}, x)$ be the equivalent and compensating risk premia for two different utility functions, $v_{1}(x)$ and $v_{2}(x)$. If $\pi_{1}(\tilde{z}, x) \geq(>) \pi_{2}(\tilde{z}, x)$ for all $x$ where both $\pi_{1}(\tilde{z}, x)$ and $\pi_{2}(\tilde{z}, x)$ exist and both exist for some value of $x$, then $\pi_{1}^{*}(\bar{z}, x) \geq(>) \pi_{2}^{*}(\tilde{z}, x)$ for all $x$ where both $\pi_{1}^{*}(\tilde{z}, x)$ and $\pi_{2}^{*}(\tilde{z}, x)$ exist. Similarly, for one utility function and two risks $\bar{z}_{1}$ and $\tilde{z}_{2}$, if $\pi\left(\tilde{z}_{1}, x\right) \geq(>) \pi\left(\tilde{z}_{2}, x\right)$ for all $x$ where both exist, and both exist for some value of $x$, then $\pi^{*}\left(\tilde{z}_{1}, x\right) \geq(>) \pi^{*}\left(\bar{z}_{2}, x\right)$ for all $x$ where both exist. Both statements are also true if the equivalent and compensating premia are interchanged.

A proof is in Appendix A. As a consequence of this lemma, not only only are equivalent and compensating risk premia approximately equal "in the small," but almost all significant qualitative results about equivalent risk premia are interchangeable with corresponding results about compensating risk premia, including the result the result about risk premia "in the large" discussed above. Furthermore, because of the close analogy between risk premia and precautionary premia, we can be confident that a result about equivalent precautionary premia will imply a corresponding result about compensating precautionary premia. ${ }^{11}$

\section{The Analogy Between Precautionary Saving and Risk Aversion}

From this point on, our discussion will be much more concrete, as we focus on the consumptionsavings decision under uncertainty as a paradigmatic example of choice under risk. Establishing the formal correspondence between precautionary saving and risk aversion requires us to set out a simple model representing this consumption-savings decision. The simplest such model is one that has only two periods, in which a consumer imbued with additively time-separable utility faces uncertain labor income in the second period. This is the model taken up here. The extension to more than two periods is difficult, but important, and is pursued in Kimball (1988b). The extension to the case of nonseparable utility is relatively straightforward, but cumbersome, and is addressed in Appendix C.

To further simplify the presentation, it will be assumed that the risk-free rate and other rates of return are exogenously fixed and that when an agent faces incomplete markets, the constraint

11 In the case of precautionary premia, the assumption of strict monotonicity and continuity in the Lemma becomes the assumption that $\frac{\partial Y(\theta, \delta)}{\partial \delta}$ is a continuous and either monotonically increasing or monotonically decreasing function of $\theta$. 
that an agent cannot borrow against more than the minimum value of his or her human wealth is never binding except at the end of the last period of the agent's existence. Since the interest rate is exogenously given, it is easiest to calculate everything in present-value terms, so that we can, without loss of generality, treat the real risk-free rate as if it were zero. Finally, it will be assumed that labor supply is inelastic, so that labor income can be treated as if it were manna from heaven. ${ }^{12}$ With the foregoing simplifications, the consumer's decision problem is:

$$
\max _{\varepsilon} u(c)+\mathbf{E} v\left(w_{0}-c+y\right)
$$

where $u$ is the first period utility function, $c$ the first period consumption, $\mathbf{E}$ an expectation conditional on first-period information, $v$ the second-period utility function, $w_{0}$ the consumer's initial assets plus his first-period labor income (which is received before the first-period consumption decision must be made) and $y$ the second-period labor income.

It will be convenient to write $y=\tilde{y}+\bar{y}$, dividing second-period labor income $y$ into its expectation $\bar{y}$ and a mean zero risky component $\bar{y}$, and to define $w=w_{0}+\bar{y}$, adding mean secondperiod income to initial assets to get the total of human and non-human wealth (which is what determines consumption in the case of certainty equivalence). We will also define for later use $s=w-c$, the amount of "saving" out of total human and non-human wealth. The consumer's decision problem can then be rewritten:

$$
\max _{c} u(c)+\mathbf{E} v(w-c+\tilde{y})
$$

The first-order condition for (7) is

$$
u^{\prime}(\mathbf{c})=\mathbf{E} v^{\prime}(w-c+\tilde{y})
$$

It is clear from this first-order condition that the risk $\tilde{y}$ in second-period income will affect consumption in the first period only insofar as it affects second-period expected marginal utility. The fact that expected marginal utility is unaffected by the addition of mean-zero risks in the case of quadratic utility is what yields certainty equivalence in that case. Even when certainty equivalence does not hold, if there is some quantity $\psi^{*}$ that can compensate for the effect of the risk $\tilde{y}$ on second-period expected marginal utility - that is, a compensating precautionary premium satisfying $v^{\prime}(w-c)=\mathbf{E} v^{\prime}\left(w-c+\tilde{y}+\psi^{*}\right)$, then first-period consumption would be unaltered by the

12 Despite the fact that labor supply is inelastic, I will continue to refer to this income as "labor income" in order to distinguish it from "capital income," which is determined endogenously. 
addition of the risk $\vec{y}$ plus the compensating precautionary premium $\psi^{*}$. Similarly, if there is an equivalent precautionary premium $\psi$ such that $y^{\prime}(w-c-\psi)=\mathbf{E} v^{\prime}(w-c+\tilde{y})$, then the elimination of the risk $\tilde{y}$ at the cost to consumer of the certain quantity $\psi$ would leave optimal first-period consumption unchanged.

Interpreting the two precautionary premia in a slightly different way, the compensating precautionary premium $\psi^{*}$ shows how far the first-period consumption function will shift to the right at a given level of consumption, since it shows how much more wealth is needed to compensate for the effect of the risk on consumption. Similarly, the equivalent precautionary premium $\psi$ shows the leftward shift of the consumption function that would result from elimination of the risk $\tilde{y}$. Appendix B contains a demonstration of these two propositions.

To place this model within the Rothschild-Stiglitz framework used in Section II, we could write

$$
V(w+\bar{y}, c)=u(c)+v(w-c+\bar{y})
$$

Consumption is the decision variable, so that $c$ takes on the role of $\delta$, while $w$ has the role of $\theta_{0}, \tilde{y}$ the role of $\tilde{\theta}$ and $w+\tilde{y}$ the role of $\theta$ itself. ${ }^{13}$ In place of $\frac{\partial V}{\partial \delta}$ we have $\frac{\partial V}{\partial c}=u^{\prime}(c)-v^{\prime}(w-c+\tilde{y})$, and in place of $\frac{\partial^{2} V}{\partial \theta \partial \delta}$ we have $-v^{\prime \prime}(w-c+\tilde{y})$, which is always positive. Because $u^{\prime}(c)$ is constant for a fixed value of the decision variable $c$, we can ignore this term in the definition of precautionary premia for this model. Moreover, the term $-v^{\prime}(w-c+\tilde{y})$ is a function of $c$ only through $w-c=s$, so that a precautionary premium for one combination of consumption and wealth is also a precautionary premium for all other combinations of consumption and wealth involving the same level of "saving" $s$ out of total expected human and nonhuman wealth. In particular, for a given value of $s$, one can search out the combination of $w$ and $c$ for which the first-order condition (8) is satisfied, allowing us to give an economic interpretation to the precautionary premia for any value of $s$.

The analogy between the theory of risk aversion and the theory of precautionary saving is particularly simple. The negative of marginal utility $-v^{\prime}$ plays substantially the same role for precautionary saving that the utility function itself plays for risk aversion. For example, concavity of $v$ indicates risk aversion, while concavity of $-v^{\prime}$ or $v^{\prime \prime \prime}(\cdot)>0$ indicates a positive precautionary saving motive. As another example, the index of absolute prudence in this model, where it represents the strength of the precautionary saving motive, is

$$
\eta(w, c)=\eta(s)=-\frac{\left(-v^{\prime}(s)^{\prime \prime}\right.}{\left(-v^{\prime}(s)\right)^{\prime}}=-\frac{v^{\prime \prime \prime}(s)}{v^{\prime \prime}(s)}
$$

13 It is because $w$ has the role of $\theta_{0}$ that the precautionary premia show up here as additions and subtractions to wealth. 
Thus, the analogy between absolute risk aversion and absolute prudence is especially obvious in this context. In general, virtually every theorem about risk aversion has an application to precautionary saving by substituting $-v^{\prime}$ for $v$ and making other appropriate adjustments. We will proceed in exactly this way: reinterpreting many of the most important theorems about risk aversion as theorems about precautionary saving. ${ }^{14}$

\section{Prudence as a Measure of the Intensity of the Precautionary Saving Motive}

Applying the results of Section II to precautionary saving, we find first that the increase in the wealth needed to induce any given level of first-period consumption-or in other words, the rightward shift of the consumption function-due to a small risk is approximately $\eta(s)=-\frac{v^{\prime \prime \prime}(s)}{v^{\prime \prime}(s)}$ (where the total "saving" out of human and nonhuman wealth $s$ is also the mean of second-period consumption) times half the variance of the risk. Similarly, the leftward shift of the consumption function due to the elimination of a small risk is approximately $\eta(s)$ times half the variance of the risk.

Second, given two second-period utility functions $v_{1}$ and $v_{2}$, if $\eta_{1}(s)=\frac{-v_{1}^{\prime \prime \prime}(s)}{v_{1}^{\prime \prime}(s)}>\frac{-v_{2}^{\prime \prime \prime}(s)}{v_{2}^{\prime \prime \prime}(s)}=\eta_{2}(s)$ for all $s$, then

$$
\psi_{1}(\tilde{y}, s)>\psi_{2}(\tilde{y}, s)
$$

for all $s$ and $\bar{y}$, and

$$
\psi_{1}^{*}(\tilde{y}, s)>\psi_{2}^{*}(\tilde{y}, s)
$$

for all $s$ and $\tilde{y}$. Therefore, for points with the same amount of saving out of total human and nonhuman wealth $s$, the risk $\bar{y}$ in second-period labor income causes a greater rightward shift of the consumption function for an individual with second-period utility $v_{1}$ than one with second-period utility $v_{2}$. If the first-period utility functions are such that the consumption functions in the absence of the income risk $\tilde{y}$ are identical (for example, if $u_{1}(c)=\frac{1}{\lambda} v_{1}(\lambda c)$ and $u_{2}(c)=\frac{1}{\lambda} v_{2}(\lambda c)$ so that the consumption functions are both linear, with slope $\frac{1}{1+\lambda}$ ) then points with the same saving and the same initial second-period consumption also have the same first-period consumption; therefore agent 1 would react to $\tilde{y}$ with a greater rightward shift of the whole curve representing the consumption function. Similarly, the leftward shift of the consumption function due to eliminating the risk $\tilde{y}$ is greater for agent 1 than for agent 2 at points with the same amount of saving $s$. If by chance

\footnotetext{
14 I am grateful to Leslie Young for suggesting this way of proceeding.
} 
the consumption functions in the face of the income risk $\tilde{y}$ are identical, ${ }^{15}$ points with the same "saving" and mean second-period consumption $s$ have the same consumption $c$ as well, so that the whole graph of the consumption function shifts further left for agent 1 than for agent 2 when the income risk is eliminated.

In addition to applying the results of Section II to precautionary saving, the simple structure of precautionary saving under additively separable utility makes it possible to bring to bear the result of Arrow (1965) that a globally more risk-averse individual will choose to invest less in a risky security. Formally, Arrow states that if $v_{\mathbf{1}}$ is globally more risk averse than $v_{2}$ (that is, $a_{1}(x)>a_{2}(x)$ for all $\left.x\right)$ then $\alpha_{1}(\tilde{z}, x)<\alpha_{2}(\tilde{z}, x)$ for all $x$, where

$$
\alpha_{1}(\tilde{z}, x)=\arg \max _{\alpha} \mathbf{E} v_{1}(x+\alpha \tilde{z})
$$

and

$$
\alpha_{2}(\tilde{z}, x)=\arg \max _{\alpha} \mathbf{E} v_{2}(x+\alpha \tilde{z})
$$

As Arrow (1965) also makes clear, differentiating twice with respect to $\alpha$ shows that expected utility is concave in $\alpha$, and therefore that expected utility monotonically increases with $\alpha$ up to the point where the maximum is reached and monotonically decreases thereafter.

To make the application of the foregoing result to precautionary saving, substitute $-v_{1}^{\prime}$ for $v_{1}$ and $-v_{2}^{\prime}$ for $v_{2}$ to find that if $\eta_{1}(s)>\eta_{2}(s)$ for all $x$, then $\beta_{1}(\tilde{y}, s)<\beta_{2}(\tilde{y}, s)$, where

$$
\beta_{1}(\tilde{y}, s)=\arg \max _{\beta} \mathbf{E}\left[-v_{1}^{\prime}(s+\beta \tilde{z})\right],
$$

and

$$
\beta_{2}(\tilde{y}, s)=\arg \max _{\beta} \mathbf{E}\left[-v_{2}^{\prime}(s+\beta z)\right]
$$

How can this be interpreted? Since

$$
-u^{\prime}(c)=\mathbf{E}\left[-v^{\prime}(s+\beta \tilde{z})\right]
$$

for an individual allowed to freely choose his or her level of consumption and saving, the level of consumption corresponding to a given level of saving must be greatest for the value of $\beta$ that maximizes the right-hand side. To look at things another way, since total human and non-human

15 Note that this is different than the consumption two consumption functions being the same in the absence of risk, so the following statement is not just a trivial consequence of the preceding one. 
wealth other than the risky asset $\beta \tilde{z}$ is equal to consumption plus saving $(w=c+s)$, we can see that the wealth corresponding to a given level of saving is maximized by a lower value of $\beta$ for agent 1 than for agent 2. Graphically, the savings function at a given level of $s$ begins to move leftward (implying an increased average propensity to save out of initial wealth) at lower values of $\beta$ for agent 1 than for agent 2 .

\section{Decreasing, Increasing, or Constant Absolute Prudence}

Using the fact that $a_{1}(x)>a_{2}(x)$ for all $x$ implies greater global risk aversion for $v_{1}$ than for $v_{2}$, it is easy to show, as Pratt (1964) does, that diminishing absolute risk aversion $(a(x)>a(x+\epsilon)$ for all $x$ and all $\epsilon>0)$ implies that the risk premium $\pi(\tilde{z}, x)$ always decreases with $x$. One need only substitute into the propositions above $v_{1}(x)=v(x)$ and $v_{2}(x)=v(x+\epsilon)$ where $\epsilon$ is any strictly positive number. One also discovers by this exercise that $\pi^{*}(\tilde{z}, x)$ is decreasing in $x$ and that the optimal amount of the risky security, $\alpha(\bar{z}, x)$ is increasing in $x$ if absolute risk aversion is decreasing. By the same kind of reasoning, $\pi(\bar{z}, x)$ and $\pi^{*}(\bar{z}, x)$ are increasing in $x$ and $\alpha(\tilde{z}, x)$ is declining in $x$ if absolute risk aversion is increasing $(a(x)<a(x+\epsilon)$ for all $x$ and all $\epsilon>0)$. Finally, $\pi(\tilde{z}, x)$, $\pi^{*}(\tilde{z}, x)$ and $\alpha(\tilde{z}, x)$ are constant in $x$ if absolute risk aversion is constant. ${ }^{16}$

The application of these results to precautionary saving follows the same pattern as before. If absolute prudence is decreasing $(\eta(s)>\eta(s+\epsilon)$ for all $s$ and all $\epsilon>0)$ then $-v^{\prime}(x)$ is a decreasing absolute risk aversion utility function and both $\psi(\tilde{y}, s)$ and $\psi^{*}(\tilde{y}, s)$ are decreasing in $s$, while $\beta(\tilde{y}, s)$ is increasing in $s$. Similarly, if absolute prudence is increasing $(\eta(s)<\eta(s+\epsilon)$ for all $s$ and all $\epsilon>0)$, then $\psi(\tilde{y}, s)$ and $\psi^{*}(\bar{y}, s)$ are increasing in $x$, while $\beta(\tilde{y}, s)$ is decreasing in s; and if absolute prudence is constant, $\psi(\tilde{y}, s), \psi^{*}(\tilde{y}, s)$ and $\beta(\tilde{y}, s)$ will be constant. ${ }^{17}$

The preceding facts about $\psi^{*}(\tilde{y}, s)$ allow us to determine the effect of income risk on the marginal propensity to consume. As shown in Appendix B, the formal statement of the fact that $\psi^{*}(\tilde{y}, s)$ is the rightward shift in the consumption function is

$$
w(c, \tilde{y})=w(c, o)+\psi^{*}(y, w(c, o)-c)
$$

where $w(c, \tilde{y})$ is the amount of wealth necessary to induce consumption $c$ in the face of the income risk $\tilde{y}$. If $\psi^{*}$ is differentiable,

$$
\frac{\partial w(c, \tilde{y})}{\partial c}=\frac{\partial w(c, 0)}{\partial c}+\frac{\partial \psi^{*}(\tilde{y}, s(c, 0))}{\partial s} \frac{\partial s(c, 0)}{\partial c},
$$

\footnotetext{
16 Note that $\pi=\pi^{*}$ if $\pi$ and $\pi^{*}$ are constant in $x$.

17 Note that $\psi=\psi^{*}$ if $\psi$ and $\psi^{*}$ are constant in $s$.
} 
where $s(c, o)=w(c, o)-c$. As a result of the strict concavity and additive separability of utility, $\frac{\partial s(c, 0)}{\partial c}>0$ (i.e., second-period consumption is a normal good), so that the effect of the income risk $\tilde{y}$ on the reciprocal of the marginal propensity to consume $\frac{\partial w}{\partial c}$ is the same as the sign of $\frac{\partial \psi^{*}}{\partial s} \cdot{ }^{18}$ It is obvious then that the effect of income risk on the marginal propensity to consume $\frac{\partial c}{\partial w}$ has the opposite sign from that of $\frac{\partial \psi^{*}}{\partial s}$. Therefore, if $\eta(s)$ is decreasing in $s$, then $\frac{\partial \psi^{*}}{\partial s}<0$ and income risk increases the marginal propensity to consume. ${ }^{19}$ If $\eta(s)$ is increasing in $s, \frac{\partial \psi^{*}}{\partial s}>0$ and income risk reduces the marginal propensity to consume. Finally if $\eta(s)$ is constant, $\psi^{*}$ is also constant and the marginal propensity to consume is unaffected by income risk.

Further discussion of the effect of income risk on the marginal propensity to consume, along with a discussion of the plausibility of the assumption of decreasing absolute risk aversion can be found in Kimball (1988b).

The facts stated above about the equivalent precautionary premium $\psi(\tilde{y}, s)$ are redundant, in the sense that looking at the equivalent precautionary premium $\psi(\bar{y}, s)$ in the context of decreasing, increasing, or constant absolute prudence can only tell us that if income risk is eliminated, the effect on the marginal propensity to consume is the opposite of what happens when income risk is introduced. More illuminating is the interpretation of the fact that $\beta(\tilde{z}, s)$ is increasing, decreasing or constant as absolute prudence is respectively decreasing, increasing or constant. It indicates, for example, that in the case of decreasing absolute prudence, the amount of the risk $\tilde{z}$ the agent can absorb before the reduction in saving due to the positive mean of $\tilde{z}$ begins to be dominated by precautionary saving effects (or more precisely, the value of $\beta$ beyond which increases in $\beta$ reduce the wealth corresponding to a given amount of saving) is increasing in $s$. In the case of increasing absolute prudence, this watershed amount of risk is decreasing in $s$; and in the case of constant absolute prudence, it is constant.

\section{The Effect of Income Risk in the Presence of Other Independent Risks}

One possible objection to the original Arrow-Pratt theory of risk aversion is that it tends to compare risky situations with situations of certainty. Actual economic agents are more likely to be comparing two situations of uncertainty. Fortunately, Kihlstrom, Romer and Williams (1981), and Nachman (1982) show that the Arrow-Pratt theory of risk aversion applies not only to risks imposed on a world of certainty, but also to risks added to the preexisting uncertainty, as long as (a)

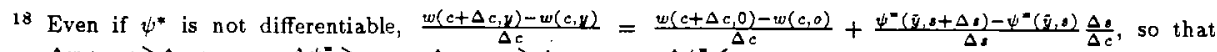

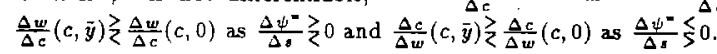

19 One special case of decreasing absolute prudence is the case of constant relative risk aversion, for which Zeldes (1986) found this effect of income risk on the marginal propensity to consume using computer simulations. 
the added risks have a probability distribution that is independent of the preexisting uncertainty and (b) the utility functions involved have decreasing absolute risk aversion. ${ }^{20}$ The implications for precautionary saving are immediately apparent: as long as the risks involved are independent of the background uncertainty and the utility functions involved have decreasing absolute prudence, all of the preceeding results about precautionary saving are true even in the presence of background: uncertainty. ${ }^{21}$

Addressing the same general issue of an agent's response to one risk in the presence of another independent risk, Pratt and Zeckhauser (1987) discuss the conditions under which being forced to face one undesirable risk will make an individual less willing to take on another, independent risk ${ }^{22}$; or equivalently, conditions under which the risk premium of two independent risks put together will be more than the sum of the risk premia of each risk taken separately. ${ }^{23}$ Pratt and Zeckhauser's most striking finding is that a broad class of utility functions have this property, including all of those that are infinitely differentiable and have derivatives alternating in sign on some semi-infinite interval $\left(x_{0}, \infty\right)$ (i.e., $v^{\prime}(x) \geq 0, v^{\prime \prime}(x) \leq 0, v^{\prime \prime \prime}(x) \geq 0, v^{\prime \prime \prime \prime}(x) \leq 0$, etc., for all $\left.x>x_{0}\right)$. This result is immediately applicable to precautionary saving since the condition of infinite differentiability and derivatives of alternating sign is one that if satisfied by $v$, is also satisfied by $-v^{\prime}$. Under this condition, satisfied by many commonly used utility functions, the effect of independent risks on precautionary saving will be more-than-additive, as measured by the precautionary premia.

\section{The Drèze-Modigliani Substitution Effect}

Drèze and Modigliani (1972), studying a two-period model similar to the model in this paper, analyze the effect of income risk on first-period income into two components. One component is the reduction in consumption one would expect by looking at the reduction in utility caused by income risk and calculating the change in consumption that would result if an equal reduction in utility had occurred as the result of a reduction in wealth. This they call the "wealth effect," though the label is somewhat confusing. The other component is the reduction in first-period consumption beyond what one would expect from looking at the reduction in utility caused by the income risk.

20 The basic theorem from which all of the other results of Kihlstrom, Romer and Williams stem is that if $v_{1}(x)$ is globally more risk averse than $v_{2}(x)$, then the derived utility function $\hat{v}_{1}(x)=E v_{1}(x+\bar{\nu})$ is globally more risk averse than $v_{2}(x)=\mathrm{E} v_{2}(x+\tilde{\nu})$ as long as either $v_{1}(x)$ or $v_{2}(x)$ has decreasing absolute risk aversion.

21 Ross (1981) shows that one cannot readily extend previous results to the case in which additional risk is a gener ai mean-preserving spread of preexisting risk rather than being distributed independently of that preexisting risk.

22 Pratt and Zeckhauser look for utility functions that guarantee this property even in the presence of a third independent background risk; thus their work meshes nicely with that of Kihlstrom, Romer and Williams (1981)

23 If it is equivalent risk premia at issue, both risks must be undesirable, but this is not necessary if it is compensating risk premia at issue. 
This they term the "substitution effect." They show that this "substitution effect" is positive if preferences for second-period consumption display decreasing absolute risk aversion, negative in the case of increasing absolute risk aversion and zero in the case of constant absolute risk aversion.

In the model presented here, the correspondence between the direction of the substitution effect and decreasing versus increasing absolute risk aversion is a simple consequence of the fact that absolute prudence is greater than or less than absolute risk aversion depending on whether absolute risk aversion is decreasing or increasing. To see this, one need only take the logarithmic derivative of absolute risk aversion:

$$
\frac{a^{\prime}(x)}{a(x)}=\frac{d}{d x} \ln \left(\frac{-v^{\prime \prime}(x)}{v^{\prime}(x)}\right)=\frac{v^{\prime \prime \prime}(x)}{v^{\prime \prime}(x)}-\frac{v^{\prime \prime}(x)}{v^{\prime}(x)}=a(x)-\eta(x) .
$$

Clearly, as long as we are dealing with risk averse functions so that $a(x)>0$, then

$$
a^{\prime}(x) \lesseqgtr 0 \text { as } \eta(x) \frac{\gtrless}{<} a(x) .
$$

Focusing on the most important case, decreasing absolute risk aversion implies that prudence exceeds risk aversion. ${ }^{24}$ To put it another way, decreasing absolute risk aversion implies that $-v^{\prime}(x)$ is more risk averse than $v(x)$.

If $-v^{\prime}(x)$ is more risk averse than $v(x)$, then precautionary saving effects are at least as large as the effects of risk aversion. In particular, given decreasing absolute risk aversion,

$$
\psi(\tilde{y}, s)>\pi(\tilde{y}, s)
$$

$$
\psi^{*}(\tilde{y}, s)>\pi^{*}(\tilde{y}, s)
$$

and

$$
\beta(\bar{z}, s)<\alpha(\bar{z}, s)
$$

where $\beta(\tilde{z}, s)$ maximizes $\mathrm{E}\left[-v^{\prime}(s+\beta \tilde{z})\right]$ and $\alpha(\tilde{z}, s)$ maximizes $\mathrm{E}[v(s+\alpha \tilde{z})]$. Furthermore, if $\tilde{y}$ is independent of $\tilde{\nu}$,

$$
\psi(\tilde{y}, s+\tilde{\nu})>\pi(\tilde{y}, s+\tilde{\nu})
$$

24 Decreasing absolute risk aversion is almost universally considered a reasonable, or even obligatory assumption since it is implied by such behavior as investing more in risky securities as one becomes wealthier. (See Pratt (1964).) 
and

$$
\psi^{*}(\tilde{y}, s+\tilde{\nu})>\pi^{*}(\tilde{y}, s+\tilde{\nu})
$$

Thus, decreasing absolute risk aversion implies that whether starting from certainty or considering additional risks independent of background uncertainty, each precautionary premium is greater than the corresponding risk premium, and the amount of a risky security an agent would freely choose is larger than the amount which, if forced on him or her, would require the most wealth to induce a given amount of saving.

We can give a more vivid interpretation of the above facts about precautionary and risk premia. $\psi^{*}>\pi^{*}$ means that the extra wealth it would take to bring the agent's consumption in the presence of $\tilde{y}$ back to what it was in the absence of $\tilde{y}$ is greater than the amount it would take to bribe the individual to accept the risk $\tilde{y}$. Therefore, if a monopolist selling the risk $\tilde{y}$ plus a certain positive quantity effectively price-discriminates so that the consumer gets no surplus from buying it (but the purchase is still voluntary), the consumer's first-period consumption will decline. (However, if the consumer gets some surplus - that is, a bigger bribe to take $\tilde{y}$ than necessary-first-period consumption may go up.) Conversely, $\psi>\pi$ means that the maximum amount a consumer is willing to pay for complete insurance, or insurance from a risk independent of other risks, is less than the amount that would have to be taken from the agent in order to keep his or her consumption the same despite the reduction in risk. Therefore, any voluntary purchase of complete insurance, or insurance from a risk independent of other risks, will increase the agent's first-period consumption; since even if the agent was indifferent about getting the insurance, consumption would increase, and whatever consumer's surplus the agent gets (i.e., however much less the agent pays than he or she is willing to pay) also tends to increase first-period consumption.

Finally, the fact that $\beta(\tilde{z}, s)<\alpha(\tilde{z}, s)$ can be interpreted graphically as in Figures 1 and 2. Figure 1 shows a graph of expected utility and expected marginal utility as a function of the amount $\lambda$ of a risky security held. Expected marginal utility is minimized, and therefore the first-period consumption and wealth corresponding to a given level of saving is maximized, for a smaller value of $\lambda$ than the one that maximizes utility. Since both $\mathrm{E}[v(s+\lambda \tilde{z})]$ and $\mathrm{E}\left[-v^{\prime}(s+\lambda \tilde{z})\right]$ are concave functions ${ }^{25}$ of $\lambda$, this means that at the amount of the risky security the agent will choose and for a range of lower amounts and all higher amounts of the risky security, more of that security will reduce the wealth needed to induce a given amount of saving, or equivalently, more of the risky

25 See Arrow (1965), or simply differentiate twice. 
security will lead to increased precautionary saving and less first-period consumption at the same level of wealth.

Figure 2 shows these facts in a different way. Decreasing absolute risk aversion implies both that the curve $\lambda=\alpha(\tilde{z}, s)$ will be upward sloping in the $\lambda$-s plane and that the curve $\lambda=\beta(\tilde{z}, s)$ will be to the left of $\lambda=\alpha(\tilde{z}, s)$. (The curve $\lambda=\beta(\tilde{z}, s)$ is upward sloping in the case of decreasing absolute prudence, but otherwise may not be.) There is a region at the left, where the amount of the risky security is low enough relative to saving that more of the security increases both expected utility and first-period consumption. At the right, the amount of the risky security is so large that if forced to hold even more, both the agent's expected utility and his or her first-period consumption would decrease. In the middle is a region where more of the risky security increases expected utility but reduces first-period consumption. On the boundary where increases in the risky security have no effect on first-period consumption (where $\lambda=\beta(\bar{z}, s)$ ), they always increase expected utility. Finally, at the amount of the risky security that maximizes expected utility $(\lambda=\alpha(\tilde{z}, s))$, more of the risky security always increases precautionary saving and decreases first-period consumption.

In view of the facts above, it can be seen that the opportunity to make risky investments has two possibly divergent effects on the marginal propensity to consume. On the one hand, free choice about risky investments may lead agents to voluntarily face more risk than they otherwise would-risk which tends to increase the marginal propensity to consume in the case of decreasing absolute prudence (or decrease it in the case of increasing absolute prudence). On the other hand, assuming we have decreasing absolute risk aversion, the endogenous increase in risky investment that comes with an increase in wealth will tend to reduce consumption because of the DrèzeModigliani substitution effect, implying a lower overall marginal propensity to consume. ${ }^{26}$ These effects are discussed in more detail in Kimball (1988b).

\section{Conclusion}

Since Leland (1968), it has been recognized that a positive third derivative of the utility function indicates a precautionary saving motive-that is, that uncertainty about future income will reduce current consumption and increase current saving. Thus, the sign of the third derivative of the utility function governs the presence or absence of a precautionary saving motive just as the sign of the second derivative governs the presence or absence of risk aversion. We have shown

26 In the case of increasing absolute risk aversion, the endogenous decrease in risky investment that comes with an increase in wealth will tend to reduce consumption, also tending to lower the overall marginal propensity to consume. In the case of constant absolute risk aversion there is no endogenous change in risky investment due to a change in wealth and so the marginal propensity to consume is unaffected by the possibility of risky investment. 
that the analogy between risk aversion and the precautionary saving motive extends much deeper. Without taking undue license, it can be said that the precautionary saving motive is risk aversion of the negative of marginal utility.

More generally, the theory of risk aversion can be used to study any situation of optimal choice under uncertainty. In particular, the Arrow-Pratt measures of absolute and relative risk aversion have the counterparts in the theory of choice under uncertainty of absolute and relative prudence, which measure the sensitivity to risk of the optimal choice of a decision variable.

Some of the specific results established about the paradigmatic case of precautionary saving are:

(1) For additively separable utility of future consumption $v$, the quantity $\frac{-v^{\prime \prime \prime}}{v^{\prime \prime}}$ is the appropriate measure of absolute prudence, and measures the strength of the precautionary saving motive, just as absolute risk aversion $\frac{-v^{\prime \prime}}{y^{t}}$ measures the strength of risk aversion.

(2) Ignoring the effects of endogenous choice of the level of risky investment, if absolute prudence, $\frac{-v^{\prime \prime \prime}}{v^{\prime \prime}}$, is decreasing, then labor income uncertainty will raise the marginal propensity to consume at any given level of consumption. Conversely, if absolute prudence is increasing, labor income uncertainty will lower the marginal propensity to consume out of wealth at a given initial level of consumption.

(3) The Dreze-Modigliani (1972) "substitution effect" can be interpreted very readily in terms of the fact the index of absolute prudence exceeds the Arrow-Pratt index of absolute risk aversion whenever absolute risk aversion is decreasing, and is less than the Arrow-Pratt measure of risk aversion when absolute risk aversion is increasing.

(4) Holding constant the initial amount of risk an agent faces, endogenous adjustment of the amount of risky security holding will result in a lower marginal propensity to consume.

(5) If the utility of future consumption $v$ is infinitely differentiable with its derivatives alternating in sign then the precautionary saving effects of independent income risks (as measured by precautionary premia) are more than additive.

(6) The main results can easily be extended to the case of independent background risk and to the case of non-additively-separable von Neumann-Morgenstern utility. ${ }^{27}$

Since uncertainty is present in almost all aspects of life, the theory of choice under uncertainty is likely to become an even more important part of economics in the future than it is now. Although it will not answer every question about choice under uncertainty, we can hope that the close analogy

\footnotetext{
27 That extension is in Appendix C.
} 
between risk aversion and the sensitivity of optimal choices to risk will continue to be fruitful in illuminating many other responses to risk as it illuminates precautionary saving.

\section{Appendix A}

\section{The Connection Between Equivalent and Compensating Risk Premia}

To see that the functions $f(x)=x-\pi(\tilde{z}, x)$ and $g(x)=x+\pi^{*}(\tilde{z}, x)$ are mutual inverses one must examine closely the the definitions of $\pi(\tilde{z}, x)$ and $\pi^{*}(\tilde{z}, x)$. If

$$
x^{*}=x-\pi(\tilde{z}, x)
$$

then by the definition of $\pi(\tilde{z}, x)$,

$$
v\left(x^{*}\right)=\mathbf{E} v(x+\bar{z})
$$

but by the definition of $\pi^{*}\left(\tilde{z}, x^{*}\right)$,

$$
v\left(x^{*}\right)=\mathbf{E} v\left(x^{*}+\tilde{z}+\pi^{*}\left(\tilde{z}, x^{*}\right)\right) .
$$

Equations (A.2) and (A.3), plus the strict monotonicity of $v$, imply that

$$
x=x^{*}+\pi^{*}\left(\tilde{z}, x^{*}\right)
$$

The monotonicity and continuity of $v$ insures that both $f(x)$ and $g(x)$ are also continuous and monotonically increasing. Portraying $f(x)$ and $g(x)$ graphically, as in Figure 3 , one can see that if $\pi_{1}(\bar{z}, x)>\pi_{2}(\bar{z}, x)$ for all $x$ so that the curve for $v_{1}$ is below the curve for $v_{2}$, then the curve for $v_{1}$ is to the right of the curve for $v_{2}$, so that $\pi_{1}^{*}\left(\bar{z}, x^{*}\right)>\pi_{2}^{*}\left(\tilde{z}, x^{*}\right)$ for all $x^{*}$, and vice versa. Similarly, if $\pi\left(\bar{z}_{1}, x\right)>\pi\left(\tilde{z}_{2}, x\right)$ for all $x$ one can show graphically that $\pi^{*}\left(\tilde{z}_{1}, x\right)>\pi^{*}\left(\bar{z}_{2}, x\right)$ for all $x$ and vice versa.

The qualifications of the Lemma in Section II arise because the equivalent and compensating risk premia may sometimes fail to exist.

We will prove the Lemma by contradiction. Defining $f_{1}, f_{2}, g_{1}$ and $g_{2}$ in the obvious manner, we know by hypothesis that there is an $x_{0}$ for which $f_{1}\left(x_{0}\right) \leq f_{2}\left(x_{0}\right)$. Now suppose there were an $x_{0}^{*}$ for which $g_{1}\left(x_{0}^{*}\right)<g_{2}\left(x_{0}^{*}\right)$. If so, either $x_{0}^{*} \leq f_{2}\left(x_{0}\right)$ or $x_{0}^{*} \geq f_{2}\left(x_{0}\right)$. 
If $x_{0}^{*} \leq f_{2}\left(x_{0}\right)$, then $g_{1}\left(x_{0}^{*}\right)<g_{2}\left(x_{0}^{*}\right) \leq x_{0}$ by the monotonicity of $g_{2}$. With $g_{2}\left(x_{0}^{*}\right)$ between two values of $x$ for which $f_{1}$ exists, the definition of $f_{1}$ insures that $f_{1}\left(g_{2}\left(x_{0}^{*}\right)\right)$ exists, and by hypothesis, we know that $f_{1}\left(g_{2}\left(x_{0}^{*}\right)\right) \leq f_{2}\left(g_{2}\left(x_{0}^{*}\right)\right)=x_{0}^{*}$. But then $f_{1}$ cannot be monotonically increasing between $g_{1}\left(x_{0}^{*}\right)$ and $g_{2}\left(x_{0}^{*}\right)$.

If $x_{0}^{*} \geq f_{2}\left(x_{0}\right)$, then by the monotonicity of $g_{1}, x_{0} \leq g_{1}\left(x_{0}^{*}\right)<g_{2}\left(x_{0}^{*}\right)$. This insures that $f_{2}\left(g_{1}\left(x_{0}^{*}\right)\right)$ exists. Then by hypothesis, $f_{2}\left(g_{1}\left(x_{0}^{*}\right)\right) \geq f_{1}\left(g_{1}\left(x_{0}^{*}\right)\right)=x_{0}^{*}$. But in that case, $f_{2}$ cannot be monotonically increasing between $g_{1}\left(x_{0}^{*}\right)$ and $g_{2}\left(x_{0}^{*}\right)$.

Therefore, there cannot exist an $x_{0}^{*}$ for which $g_{1}\left(x_{0}^{*}\right)<g_{2}\left(x_{0}^{*}\right)$; whenever both $g_{1}\left(x^{*}\right)$ and $g_{2}\left(x^{*}\right)$ both exist, $g_{1}\left(x^{*}\right) \geq g_{2}\left(x^{*}\right)$.

It is easy to modify this proof to show that the Lemma is true when stated with strict inequalities and to take care of the case of two different risks instead of two different utility functions.

\section{Appendix B}

The Connection Between Precautionary Premia and Horizontal Shifts of the Consumption Function

Let the function $c(w, \bar{y})$ be the consumption function defined by

$$
c(w, \tilde{y})=\arg \max _{c} u(c)+\mathbf{E} v(w-c+\tilde{y}),
$$

and let $w(c, \tilde{y})$ be the inverse consumption function (giving the wealth necessary to induce a given consumption level) defined by

$$
w(c(x, \tilde{y}), \tilde{y})=x
$$

Then in the case of the compensating precautionary premium, we want to show that

$$
w(c, \tilde{y})=w(c, o)+\psi^{m}(\tilde{y}, w(c, o)-c)
$$

Using the definition of $w(c, \tilde{y})$, we have

$$
u^{\prime}(c)=\mathbf{E} v^{\prime}(w(c, \tilde{y})-c+\tilde{y})=v^{\prime}(w(c, o)-c)
$$

From Table 1, the definition of $\psi^{*}(\tilde{y}, w(c, o)-c)$ is

$$
\mathrm{E} v^{\prime}\left(w(c, o)-c+\tilde{y}+\psi^{*}(\tilde{y}, w(c, o)-c)\right)=v^{\prime}(w(c, o)-c) .
$$


Together with (B.4) this implies

$$
\mathbf{E} v^{\prime}\left(w(c, o)-c+\bar{y}+\psi^{*}(\tilde{y}, w(c, o)-c)\right)=\mathbf{E} v^{\prime}(w(c, \tilde{y})-c+\tilde{y}) .
$$

Because of the strictly decreasing marginal utility of $v$, (B.6) implies (B.3).

Similarly, in the case of the equivalent precautionary premium, we want to show that

$$
w(c, o)=w(c, \tilde{y})-\psi(\tilde{y}, w(c, \tilde{y})-c) .
$$

The definition of $\psi(\bar{y}, w(c, \bar{y})-c)$, combined with (B.4), yields

$$
\begin{aligned}
v^{\prime}(w(c, \tilde{y})-c-\psi(\tilde{y}, w(c, \tilde{y})-c)) & =\mathbf{E} v^{\prime}(w(c, \tilde{y})-c+\tilde{y}) \\
& =v^{\prime}(w(c, o)-c)
\end{aligned}
$$

which, as a result of the fact that $v^{\prime}$ is strictly decreasing, implies (B.7).

\section{Appendix C}

\section{The Case of Non-Additively-Separable von Neumann-Morgenstern Utility}

In the case of non-additively-separable utility with all the other simplifications of the text maintainted, we can write

$$
V(w, c)=U(c, w-c)
$$

where $U$ is the direct utility function with consumption in the first and second periods as its two arguments. In this appendix, we can use subscripts to denote partial derivatives without confusion. Thus, in terms of the indirect utility function $V$, the consumer's optimization problem under certainty is just

$$
\max _{c} V(w, c)
$$

which has the first-order condition

$$
V_{c}(w, c)=0
$$

The cross-partial $V_{c w}$ will be positive at least for pairs of $c$ and $w$ on the consumption function if first-period consumption is a normal good, since by the implicit function theorem $\frac{d c}{d w}=-\frac{V_{c w}(w, c)}{V_{c c}(w, c)}$. 
Positivity of the denominator away from values of $c$ and $w$ on the consumption function is more difficult to guarantee, but seems likely if first-period consumption is a normal good at any interest rate and in the face of any random distribution of second-period income. The exact conditions (short of $U_{12} \geq 0$ ) needed to insure positivity of the denominator are a subject for future research.

The results of Section II can be applied straightforwardly to the nonseparable case. A simple modification of the proof in Appendix B can be used to show that for values of $c$ and $w$ and probability distributions of $\tilde{y}$ that make $\mathbf{E} V_{c}(w+\tilde{y}, c)$ equal to zero, the equivalent precautionary premium is the leftward shift of the consumption function that would follow upon removal of the income risk $\bar{y}$. Similarly, for values of $c$ and $w$ that make $V_{c}(w, c)$ equal to zero, this is the rightward shift in the consumption function due to the addition of the income risk $\tilde{y}$. The appropriate measure of absolute prudence in the nonseparable case is

$$
\eta(w, c)=\frac{-V_{c w w}(w, c)}{V_{c w}(w, c)}=-\frac{U_{122}(c, w-c)-U_{222}(c, w-c)}{U_{12}(c, w-c)-U_{22}(c, w-c)} .
$$

The relation between decreasing or increasing absolute prudence and the marginal propensity to consume can be extended to the nonseparable case if $\eta(w, c)$ is decreasing, increasing or constant when $c$ and $w$ increase in the same proportion as they do at the point on the initial consumption function with consumption equal to $c$. Normality of first-and second-period consumption could b $\epsilon$ helpful in yielding such a condition, since the right-hand side of (C.4) can be viewed naturally as a function of $c$ and $w-c$.

Results about the values of $\beta$ that maximize $\mathrm{E} V_{c}(w+\beta \tilde{z}, c)$ are difficult to interpret in the nonseparable case, since an optimal consumption choice is involved only when these expected values are zero. The results of Section VI about additional independent risks can be applied with little change to the nonseparable case, as long as the necessary conditions are satisfied by $V_{c}(w, c)$ thought of as a function of $w$.

The analysis of the Drèze-Modigliani substitution effect in the nonseparable case requires some explanation. Drèze and Modigliani (1972) do address the nonseparable case, but it is worthwhile to draw the connection between their results and the measure of prudence given above.

In the nonseparable case one must be careful in defining what one means by "decreasing (or increasing) absolute risk aversion." The expression $\frac{-V_{w w}}{V_{w}}=\frac{-U_{22}}{U_{2}}$ shows the extent of risk aversior for small risks, since the uncertainty directly affects second-period consumption, and by the envelops theorem, the adjustments in consumption due to risk will have only second-order effects on utility for small risks. For large risks the effect on utility of these adjustments in consumption must bt taken into account. The kind of "decreasing absolute risk aversion" needed to imply that prudenct 
exceeds risk aversion is that

$$
\frac{\partial}{\partial c} \frac{-V_{w w}}{V_{w}}>0
$$

which Drèze and Modigliani term "endogenously decreasing absolute risk aversion." By using the fact that the logarithmic derivative is also greater than zero, it is easy to show that this implies that

$$
\frac{V_{c w w}}{V_{w w}}>\frac{V_{c w}}{V_{w}}
$$

and assuming that $V_{c w}>0$, that

$$
\frac{-V_{c w w}}{V_{c w}}>\frac{-V_{w w}}{V_{w}}
$$

or in words, that prudence is greater than risk aversion. Similarly, if we have "endogenously increasing risk aversion," prudence will be smaller than risk aversion. The consequences of these facts for the relative sizes of risk and precautionary premia are virtually identical to the consequences of decreasing or increasing absolute risk aversion in the additively separable case, as can be seen from Drèze and Modigliani's results. Furthermore, just as in the additively-separable case, at the amount of a risky security that maximizes expected utility, an increase in the amount held will tend to reduce first-period consumption if absolute risk aversion is (endogenously) decreasing and will tend to increase first-period consumption if absolute risk aversion is (endogenously) increasing, making endogenous adjustment of investment in a risky security reduce the marginal propensity to consume in the nonseparable case as well. 


\section{References}

Arrow, Kenneth J. (1965): "Aspects of a Theory of Risk Bearing," Yrjo Jahnsson Lectures, Helsinki. Reprinted in Essays in the Theory of Risk Bearing (1971), Chicago: Markham Publishing Co. Bernoulli, Daniel (1738): "Specimen theoriae novae de mensura sortis," Comentarii Academiae Scientiarum Imperiales Petropolitanae, Vol. 5, 175-192. Translated by L. Sommer as "Exposition of a New Theory on the Measurement of Risk," Econometrica (1954), 12, 23-36.

Diamond, Peter A. and Joseph E. Stiglitz (1974): "Increases in Risk and Risk Aversion," Journal of Economic Theory, 8, 337-360.

Drèze, Jacques H., and Franco Modigliani (1972): "Consumption Decisions under Uncertainty," Journal of Economic Theory, 5, 308-335.

Feller, William (1971): An Introduction to Probability Theory and its Applications, Vol. II, 2nd Edition, John Wiley \& Sons, Inc., New York, 439-442.

Kihlstrom, Richard E., David Romer, and Steve Williams (1981): "Risk Aversion with Random Initial Wealth," Econometrica, 49, 911-920.

Kimball, Miles S. (1987): Essays on Intertemporal Household Choice, unpublished Ph. D. dissertation (Harvard University, Cambridge, MA), 100-195.

Kimball, Miles S. (1988a): "The Effect of Demand Uncertainty on a Precommitted Monopoly Price," mimeo, 1988a.

Kimball, Miles S. (1988b): "Precautionary Saving and the Marginal Propensity to Consume," mimeo, 1988b.

Kimball, Miles S. and N. Gregory Mankiw (1987): "Precautionary Saving and the Timing of Taxes," mimeo, 1987.

Leland, Hayne E. (1968): "Saving and Uncertainty: The Precautionary Demand for Saving," Quarterly Journal of Economics, 82, 465-473.

Merton, R. (1971): "Optimum Consumption and Portfolio Rules in a Continuous Time Model," Journal of Economic Theory, 373-413.

Nachman, D. C. (1982): "Preservation of 'More Risk Averse' Under Expectations," Journal of Economic Theory 28, 361-368.

Pope, Rulon D. and Jean-Paul Chavas (1985): "Producer Surplus and Risk," Quarterly Journal of Economics, 100, 85.3-870.

Pratt, John W. (1964): "Risk Aversion in the Small and in the Large," Econometrica, 32, 122-136.

Pratt, John W. and Zeckhauser, Richard J. (1987): "Proper Risk Aversion," Econometrica, January 
55, $143-154$.

Ross, Stephen A. (1981): "Some Stronger Measures of Risk Aversion in the Small and the Large with Applications," Econometrica, 49, 621-638.

Rothschild, Michael and Joseph E. Stiglitz (1970): "Increasing Risk I: A Definition," Journal of Economic Theory, 2, 225-243.

Rothschild, Michael and Joseph E. Stiglitz (1971): "Increasing Risk II: Its Economic Consequences," Journal of Economic Theory, 3, 66-84.

Sandmo, Agnar (1970): "The Effect of Uncertainty on Saving Decisions," Review of Economic Studies, 37, 353-360.

Zeldes, Stephen P. (1986): "Optimal Consumption with Stochastic Income: Deviations from Certainty Equivalence," mimeo, University of Pennsylvania, 1986. 